\title{
A way to obtain 2-uninorm on bounded lattice from uninorms defined on subintervals of bounded lattice
}

\author{
Umit Ertugrul \\ Department of Mathematics, Karadeniz Technical University, Trabzon, Turkey \\ Received: 31 January 2017, Accepted: 28 February 2017 \\ Published online: 3 April 2017.
}

\begin{abstract}
In this paper, a way to obtain 2-uninorm on bounded lattice from $U_{1}$ disjunctive uninorm on $[0, k]$ and $U_{2}$ conjunctive uninorm on $[k, 1]$ is presented. When the conditions disjunctive of $U_{1}$ or conjunctive of $U_{2}$ drop, it is showed that this method is invalid. Additionally, some properties of this construction method are investigated.
\end{abstract}

Keywords: Uninorm, 2-uninorm, bounded lattice, disjunctive, conjunctive.

\section{Introduction}

Uninorms have attracted great interest because of applications of uninorm like fuzzy logic, expert systems, neural networks, fuzzy system modelling [8,13]; after being defined on the unit interval [0,1] by Yager and Rybalov [12]. Since bounded lattice case is more complex, uninorms on bounded lattices has been a challenging problem for many researchers [3,5,6,7,11]. Besides uninorm on [0,1], 2-uninorms are defined and studied [1,2,4].

2-uninorms are special operators since they covers uninorms and nullnorms. Because of this reason some characterization of 2-uninorms on unit reel interval is done [2]. And also, some properties of 2-uninorms on unit reel interval are studied $[1,4]$. Despite being worked on unit reel interval, there is no work for 2-uninorms on bounded lattice.

In this study, a way to obtain 2-uninorm in $U_{k(e, f)}$ on bounded lattice from disjunctive uninorm $[0, k]$ and conjunctive uninorm on $[k, 1]$ is presented. If the conditions of disjunctive of $U_{1}$ or conjunctive of $U_{2}$ are removed, an example is given to show that the proposition is invalid. Under this construction method, it is showed that $k$ is absorbing element of $U^{2}$ and $U^{2}$ is neither disjunctive nor conjunctive 2-uninorm on $L$. Additionally it is obtained that even if $U_{1}$ and $U_{2}$ are idempotent, $U^{2}$ may not be idempotent 2-uninorm on $L$.

The paper is organized as follows. We shortly recall some basic notions and results in Section 2. In Section 3, we give a method to obtain 2-uninorm $U^{2} \in U_{k(e, f)}$ on bounded lattice $L$ using disjunctive uninorm on $[0, k]$ and conjunctive uninorm on $[k, 1]$. Some properties of this construction method are also investigated in Section 3.

\section{Notations, definitions and a review of previous results}

A bounded lattice $(L, \leqslant)$ is a lattice which has the top and bottom elements, which are written as 1 and 0 , respectively, i.e., there exist two elements $1,0 \in L$ such that $0 \leqslant x \leqslant 1$, for all $x \in L$. 
Definition 1. [3] Given a bounded lattice $(L, \leq, 0,1)$, and $a, b \in L$, if $a$ and $b$ are incomparable, in this case we use the notation $a \| b$.

Definition 2. [3] Given a bounded lattice $(L, \leq, 0,1)$, and $a, b \in L, a \leq b$, a subinterval $[a, b]$ of $L$ is a sublattice of $L$ defined as

$$
[a, b]=\{x \in L \mid a \leq x \leq b\} .
$$

Similarly, $(a, b]=\{x \in L \mid a<x \leq b\},[a, b)=\{x \in L \mid a \leq x<b\}$ and $(a, b)=\{x \in L \mid a<x<b\}$.

Definition 3. [11] Let $(L, \leq, 0,1)$ be a bounded lattice. An operation $U: L^{2} \rightarrow L$ is called a uninorm on $L$, if it is commutative, associative, increasing with respect to the both variables and has a neutral element $e \in L$.

In this study, the notation $\mathscr{U}(e)$ will be used for the set of all uninorms on $L$ with neutral element $e \in L$.

If $U(0,1)=0, U$ is called conjunctive uninorm and if $U(0,1)=1, U$ is called disjunctive uninorm.

If $U(x, x)=x$ for all elements $x \in L, U$ is called idempotent uninorm.

Consider the set $\mathscr{U}$ of all uninorms on $L$ with the following order. For $U, V \in \mathscr{U}$,

$$
U \leqslant V \Longleftrightarrow U(x, y) \leqslant V(x, y) \text { for all }(x, y) \in L^{2}
$$

Corollary 1. [11] Let $(L, \leq, 0,1)$ be a bounded lattice and $e \in L \backslash\{0,1\}$. Then the following uninorms $U_{T_{\wedge}}: L^{2} \rightarrow L$ and $U_{S_{\vee}}: L^{2} \rightarrow L$, respectively, are the greatest and the smallest uninorm on $L$ with neutral element $e$.

$$
\begin{aligned}
& U_{T_{\wedge}}(x, y)= \begin{cases}x \wedge y, & \text { if }(x, y) \in[0, e]^{2} \\
x \vee y, & \text { if }(x, y) \in[0, e] \times(e, 1] \cup(e, 1] \times[0, e] \\
y, & \text { if } x \in[0, e], y \| e \\
x, & \text { if } y \in[0, e], x \| e \\
1, & \text { otherwise, }\end{cases} \\
& U_{S_{\vee}}(x, y)= \begin{cases}x \vee y, & \text { if }(x, y) \in[e, 1]^{2} \\
x \wedge y, & \text { if }(x, y) \in[0, e) \times[e, 1] \cup[e, 1] \times[0, e) \\
y, & \text { if } x \in[e, 1], y \| e \\
x, & \text { if } y \in[e, 1], x \| e \\
0, & \text { otherwise. }\end{cases}
\end{aligned}
$$

Definition 4. [5] An operation $T(S)$ on a bounded lattice $L$ is called a triangular norm (triangular conorm) if it is commutative, associative, increasing with respect to the both variables and has a neutral element $1(0)$. Let $(L, \leq, 0,1)$ be a bounded lattice, $U \in \mathscr{U}(e)$ and $e \in L$. It is known that if it is $e=1$, uninorm $U$ coincides $t$-norm and if it is $e=0$, uninorm $U$ coincides $t$-conorm on $L$.

Definition 5. [10] Let $(L, \leq, 0,1)$ be a bounded lattice. An operation $V: L^{2} \rightarrow L$ is called a nullnorm on $L$, if it is commutative, associative, increasing with respect to the both variables and there is an element $a \in L$ such that $V(x, 0)=x$ for all $x \leq a, V(x, 1)=x$ for all $x \geq a$. It can be easily obtained that $V(x, a)=$ a for all $x \in L$. So, the element $a \in L$ that provide $V(x, a)=$ a for all $x \in L$ is called (absorbing) zero element for operator $V$ on $L$.

Definition 6. [4] Let $(L, \leq, 0,1)$ be a bounded lattice. An operator $F: L^{2} \rightarrow L$ is called 2-uninorm if it is commutative, associative, increasing with respect to both variables and fulfilling

$$
\forall x \leq k F(e, x)=x \text { and } \forall x \geq k F(f, x)=x,
$$


where $e, k, f \in L$ with $0 \leq e \leq k \leq f \leq 1$. By $U_{k(e, f)}$ we denote the class of all 2-uninorms on bounded lattice L. Conjunctive, disjunctive or idempotent 2-uninorm can be defined as defined for uninorms.

\section{A way to obtain 2-uninorm on bounded lattice}

In this section, a method has been proposed for generating 2-uninorm $U^{2} \in U_{k(e, f)}$ on bounded lattice $L$ using $U_{1}$ disjunctive uninorm on $[0, k]$ and $U_{2}$ conjunctive uninorm on $[k, 1]$. Even if one of conditions $U_{1}$ disjunctive uninorm on $[0, k]$ and $U_{2}$ conjunctive uninorm on $[k, 1]$ is removed, an example is given to show that the proposition may be invalid.

Proposition 1. Let $(L, \leq, 0,1)$ be a bounded lattice, $U_{1}:[0, k]^{2} \rightarrow[0, k]$ be a disjunctive uninorm with neutral element e and $U_{2}:[k, 1]^{2} \rightarrow[k, 1]$ be a conjunctive uninorm with neutral element $f$. Then, $U_{1}(x, k)=k$ for all $x \in[0, k]$ and $U_{2}(y, k)=k$ for all $y \in[k, 1]$.

Proof. Let $(L, \leq, 0,1)$ be a bounded lattice. Since $U_{1}:[0, k]^{2} \rightarrow[0, k]$ be a disjunctive uninorm with neutral element $e$, $U_{1}(0, k)=k$. Then,

$$
k=U_{1}(0, k) \leq U_{1}(x, k) \leq U_{1}(1, k)=k
$$

for all $x \in[0, k]$. Then, it is obtained that $U_{1}(x, k)=k$ for all $x \in[0, k]$. Since $U_{2}:[k, 1]^{2} \rightarrow[k, 1]$ be a conjunctive uninorm with neutral element $f, U_{2}(k, 1)=k$. Then,

$$
k=U_{2}(k, k) \leq U_{2}(y, k) \leq U_{2}(1, k)=k
$$

for all $y \in[k, 1]$. Then, it is obtained that $U_{2}(y, k)=k$ for all $y \in[k, 1]$.

Theorem 1. Let $(L, \leq, 0,1)$ be a bounded lattice, $U_{1}:[0, k]^{2} \rightarrow[0, k]$ be a disjunctive uninorm with neutral element e and $U_{2}:[k, 1]^{2} \rightarrow[k, 1]$ be a conjunctive uninorm with neutral element $f$. Then, the function $U^{2}: L^{2} \rightarrow L$ given by

$$
U^{2}(x, y)= \begin{cases}U_{1}(x, y), & \text { if }(x, y) \in[0, k]^{2} \\ U_{2}(x, y), & \text { if }(x, y) \in[k, 1]^{2} \\ k, & \text { otherwise, }\end{cases}
$$

is 2-uninorm in $U_{k(e, f)}$.

Proof. (i) Monotonicity: We prove that if $x \leqslant y$ then for all $z \in L, U^{2}(x, z) \leqslant U^{2}(y, z)$. The proof is split into all possible cases.

Let $x \leqslant k$.

1.1. $y \leqslant k$,

1.1.1. $z \leqslant k$,

$$
U^{2}(x, z)=U_{1}(x, z) \leqslant U_{1}(y, z)=U^{2}(y, z)
$$

1.1.2. $z \geq k$ or $z \| k$

$$
U^{2}(x, z)=k=U^{2}(y, z)
$$

1.2. $y \geq k$,

1.2.1. $z \leqslant k$,

$$
U^{2}(x, z)=U_{1}(x, z) \leqslant U_{1}(k, k)=k=U^{2}(y, z)
$$

1.2.2. $z \geq k$,

$$
U^{2}(x, z)=k=U_{2}(y, z)=U^{2}(y, z) .
$$


1.2.3. $z \| k$,

$$
U^{2}(x, z)=k=U^{2}(y, z)
$$

1.3. $y \| k$,

1.3.1. $z \leqslant k$,

$$
U^{2}(x, z)=U_{1}(x, z) \leqslant U_{1}(k, k)=k=U^{2}(y, z)
$$

1.3.2. $z \geq k$ or $z \| k$,

$$
U^{2}(x, z)=k=U^{2}(y, z)
$$

2. Let $x \geq k$ Then $y \geq k$.

2.1. $y \geq k$,

2.1.1. $z \leq k$ or $z \| k$,

$$
U^{2}(x, z)=k=U^{2}(y, z)
$$

2.1.2. $z \geq k$,

$$
U^{2}(x, z)=U_{2}(x, z) \leqslant U_{2}(k, k)=k=U^{2}(y, z)
$$

3. Let $x \| k$. Then $y \geq k$ or $y \| k$.

3.1. $y \geq k$,

3.1.1. $z \leq k$ or $z \| k$,

$$
U^{2}(x, z)=k=U^{2}(y, z)
$$

3.1.2. $z \geq k$,

$$
U^{2}(x, z)=k=U_{2}(k, k) \leq U_{2}(y, z)=U^{2}(y, z)
$$

3.2. $y \| k$,

3.2.1. $z \in L$,

$$
U^{2}(x, z)=k=U^{2}(y, z)
$$

(ii) Associativity. We demonstrate that $U^{2}\left(x, U^{2}(y, z)\right)=U^{2}\left(U^{2}(x, y), z\right)$ for all $x, y, z \in L$. Again the proof is split into all possible cases considering the relationships of the elements $x, y, z$ and $k$.

1. Let $x \leqslant k$.

1.1. $y \leqslant k$,

1.1.1. $z \leqslant k$,

$$
U^{2}\left(x, U^{2}(y, z)\right)=U^{2}\left(x, U_{1}(y, z)\right)=U_{1}\left(x, U_{1}(y, z)\right)=U_{1}\left(U_{1}(x, y), z\right)=U_{1}\left(U^{2}(x, y), z\right)=U^{2}\left(U^{2}(x, y), z\right)
$$

1.1.2. $z \geq k$,

$$
U^{2}\left(x, U^{2}(y, z)\right)=U^{2}(x, k)=U_{1}(x, k)=k=U^{2}\left(U_{1}(x, y), z\right)=U^{2}\left(U^{2}(x, y), z\right)
$$

1.1.3. $z \| k$,

$$
U^{2}\left(x, U^{2}(y, z)\right)=U^{2}(x, k)=U_{1}(x, k)=k=U_{1}\left(U_{1}(x, y), z\right)=U^{2}\left(U_{1}(x, y), z\right)=U^{2}\left(U^{2}(x, y), z\right)
$$

1.2. $y \geq k$,

1.2.1. $z \leqslant k$,

$$
U^{2}\left(x, U^{2}(y, z)\right)=U^{2}(x, k)=U_{1}(x, k)=k=U_{1}(k, z)=U^{2}(k, z)=U^{2}\left(U^{2}(x, y), z\right)
$$


1.2.2. $z \geq k$,

$$
U^{2}\left(x, U^{2}(y, z)\right)=U^{2}\left(x, U_{2}(y, z)\right)=k=U_{2}(k, z)=U^{2}(k, z)=U^{2}\left(U^{2}(x, y), z\right)
$$

1.2.3. $z \| k$

$$
U^{2}\left(x, U^{2}(y, z)\right)=U^{2}(x, k)=U_{1}(x, k)=k=U^{2}(k, z)=U^{2}\left(U^{2}(x, y), z\right)
$$

1.3. $y \| k$,

1.3.1. $z \leqslant k$,

$$
U^{2}\left(x, U^{2}(y, z)\right)=U^{2}(x, k)=U_{1}(x, k)=k=U_{1}(k, z)=U^{2}(k, z)=U^{2}\left(U^{2}(x, y), z\right)
$$

1.3.2. $z \geq k$,

$$
U^{2}\left(x, U^{2}(y, z)\right)=U^{2}(x, k)=U_{1}(x, k)=k=U_{2}(k, z)=U^{2}(k, z)=U^{2}\left(U^{2}(x, y), z\right)
$$

1.3.3. $z \| k$

$$
U^{2}\left(x, U^{2}(y, z)\right)=U^{2}(x, k)=U_{1}(x, k)=k=U^{2}(k, z)=U^{2}\left(U^{2}(x, y), z\right)
$$

2. Let $x \geq k$.

2.1. $y \leqslant k$,

2.1.1. $z \leqslant k$,

$$
U^{2}\left(x, U^{2}(y, z)\right)=U^{2}\left(x, U_{1}(y, z)\right)=k=U_{1}(k, z)=U^{2}(k, z)=U^{2}\left(U^{2}(x, y), z\right)
$$

2.1.2. $z \geq k$,

$$
U^{2}\left(x, U^{2}(y, z)\right)=U^{2}(x, k)=U_{2}(x, k)=k=U_{2}(k, z)=U^{2}(k, z)=U^{2}\left(U^{2}(x, y), z\right)
$$

2.1.3. $z \| k$,

$$
U^{2}\left(x, U^{2}(y, z)\right)=U^{2}(x, k)=U_{2}(x, k)=k=U^{2}(k, z)=U^{2}\left(U^{2}(x, y), z\right)
$$

2.2. $y \geq k$,

2.2.1. $z \leqslant k$ or $z \| k$,

$$
U^{2}\left(x, U^{2}(y, z)\right)=U^{2}(x, k)=U_{2}(x, k)=k=U^{2}\left(U_{2}(x, y), z\right)=U^{2}\left(U^{2}(x, y), z\right)
$$

2.2.2. $z \geq k$,

$$
U^{2}\left(x, U^{2}(y, z)\right)=U^{2}\left(x, U_{2}(y, z)\right)=U_{2}\left(x, U_{2}(y, z)\right)=U_{2}\left(U_{2}(x, y), z\right)=U_{2}\left(U^{2}(x, y), z\right)=U^{2}\left(U^{2}(x, y), z\right)
$$

2.3. $y \| k$,

$2.3 .1 z \leqslant k$,

$$
U^{2}\left(x, U^{2}(y, z)\right)=U^{2}(x, k)=U_{2}(x, k)=k=U_{1}(k, z)=U^{2}(k, z)=U^{2}\left(U^{2}(x, y), z\right)
$$


2.3.2. $z \geq k$,

$$
U^{2}\left(x, U^{2}(y, z)\right)=U^{2}(x, k)=U_{2}(x, k)=k=U_{2}(k, z)=U^{2}(k, z)=U^{2}\left(U^{2}(x, y), z\right)
$$

2.3.3z $z k$

$$
U^{2}\left(x, U^{2}(y, z)\right)=U^{2}(x, k)=U_{2}(x, k)=k=U^{2}(k, z)=U^{2}\left(U^{2}(x, y), z\right)
$$

3. Let $x \| k$.

3.1. $y \leqslant k$,

3.1.1. $z \leqslant k$,

$$
U^{2}\left(x, U^{2}(y, z)\right)=U^{2}\left(x, U_{1}(y, z)\right)=k=U_{1}(k, z)=U^{2}(k, z)=U^{2}\left(U^{2}(x, y), z\right)
$$

3.1.2. $z \geq k$,

$$
U^{2}\left(x, U^{2}(y, z)\right)=U^{2}(x, k)=k=U_{2}(k, z)=U^{2}(k, z)=U^{2}\left(U^{2}(x, y), z\right)
$$

3.1.3. $z \| k$,

$$
U^{2}\left(x, U^{2}(y, z)\right)=U^{2}(x, k)=k=U^{2}(k, z)=U^{2}\left(U^{2}(x, y), z\right)
$$

3.2. $y \geq k$,

3.2.1. $z \leqslant k$,

$$
U^{2}\left(x, U^{2}(y, z)\right)=U^{2}(x, k)=k=U_{1}(k, z)=U^{2}(k, z)=U^{2}\left(U^{2}(x, y), z\right)
$$

3.2.2. $z \geq k$,

$$
U^{2}\left(x, U^{2}(y, z)\right)=U^{2}\left(x, U_{2}(y, z)\right)=k=U_{2}(k, z)=U^{2}(k, z)=U^{2}\left(U^{2}(x, y), z\right)
$$

3.2.3. $z \| k$,

$$
U^{2}\left(x, U^{2}(y, z)\right)=U^{2}(x, k)=k=U^{2}(k, z)=U^{2}\left(U^{2}(x, y), z\right)
$$

3.3. $y \| k$,

3.3.1. $z \leqslant k$,

$$
U^{2}\left(x, U^{2}(y, z)\right)=U^{2}(x, k)=k=U_{1}(k, z)=U^{2}(k, z)=U^{2}\left(U^{2}(x, y), z\right)
$$

3.3.2. $1>z>e$,

$$
U^{2}\left(x, U^{2}(y, z)\right)=U^{2}(x, k)=k=U_{2}(k, z)=U^{2}(k, z)=U^{2}\left(U^{2}(x, y), z\right)
$$

3.3.3. $z \| e$,

$$
U^{2}\left(x, U^{2}(y, z)\right)=U^{2}(x, k)=k=U^{2}(k, z)=U^{2}\left(U^{2}(x, y), z\right)
$$

It is trivial to see the commutativity and the fact that $U^{2} \in U_{k(e, f)}$. 
Remark. If $U_{1}$ is not disjunctive or $U_{2}$ is not conjunctive, (3) may not produce a uninorm $L$. Consider the lattice $(L, \leq, 0,1)$ whose lattice diagram is displayed in Fig 1.

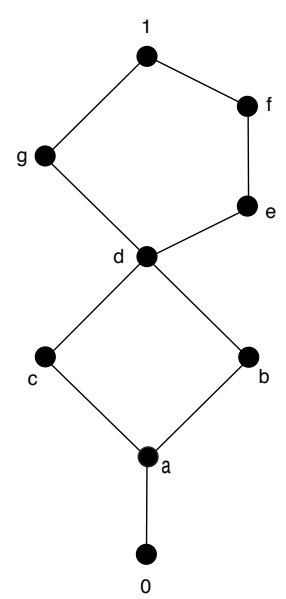

Fig. 1: $(L, \leq)$.

The function $U_{1}$ on $[0, d]$ as follows.

\begin{tabular}{|l|l|l|l|l|l|}
\hline$U_{1}$ & 0 & $a$ & $b$ & $c$ & $d$ \\
\hline 0 & 0 & 0 & $b$ & $c$ & $d$ \\
\hline$a$ & 0 & $a$ & $b$ & $c$ & $d$ \\
\hline$b$ & $b$ & $b$ & $b$ & $d$ & $d$ \\
\hline$c$ & $c$ & $c$ & $d$ & $c$ & $d$ \\
\hline$d$ & $d$ & $d$ & $d$ & $d$ & $d$ \\
\hline
\end{tabular}

Table 1: The uninorm $U_{1}$ on $[0, d]$.

and the function $U_{2}$ on $[d, 1]$ as follows.

\begin{tabular}{|l|l|l|l|l|l|}
\hline$U_{2}$ & $d$ & $e$ & $f$ & $g$ & 1 \\
\hline$d$ & $d$ & $d$ & $d$ & $g$ & 1 \\
\hline$e$ & $d$ & $d$ & $e$ & $g$ & 1 \\
\hline$f$ & $d$ & $e$ & $f$ & $g$ & 1 \\
\hline$g$ & $g$ & $g$ & $g$ & 1 & 1 \\
\hline 1 & 1 & 1 & 1 & 1 & 1 \\
\hline
\end{tabular}

Table 2: The uninorm $U_{2}$ on $[d, 1]$.

It is clear that the function $U_{1}$ is an uninorm on $[0, d]$ with neutral element $a$ and $U_{2}$ is an uninorm on $[d, 1]$ with neutral element $f$. It can be seen that $U_{1}$ is a disjunctive uninorm and $U_{2}$ is not a conjunctive uninorm. $U^{2}$ is obtained from (1) as follows. 


\begin{tabular}{|l|l|l|l|l|l|l|l|l|l|}
\hline$U$ & 0 & $a$ & $b$ & $c$ & $d$ & $e$ & $f$ & $g$ & 1 \\
\hline 0 & 0 & 0 & $b$ & $c$ & $d$ & $d$ & $d$ & $d$ & $d$ \\
\hline$a$ & 0 & $a$ & $b$ & $c$ & $d$ & $d$ & $d$ & $d$ & $d$ \\
\hline$b$ & $b$ & $b$ & $b$ & $d$ & $d$ & $d$ & $d$ & $d$ & $d$ \\
\hline$c$ & $c$ & $c$ & $d$ & $c$ & $d$ & $d$ & $d$ & $d$ & $d$ \\
\hline$d$ & $d$ & $d$ & $d$ & $d$ & $d$ & $d$ & $d$ & $g$ & 1 \\
\hline$e$ & $d$ & $d$ & $d$ & $d$ & $d$ & $d$ & $e$ & $g$ & 1 \\
\hline$f$ & $d$ & $d$ & $d$ & $d$ & $d$ & $e$ & $f$ & $g$ & 1 \\
\hline$g$ & $d$ & $d$ & $d$ & $d$ & $g$ & $g$ & $g$ & 1 & 1 \\
\hline 1 & $d$ & $d$ & $d$ & $d$ & 1 & 1 & 1 & 1 & 1 \\
\hline
\end{tabular}

Table 3: $U^{2}$ on $L$.

Since $U_{2}$ dont satisfies the conditions of Theorem $2, U^{2}$ is not an 2-uninorm on $L$ since

$$
U^{2}\left(U^{2}(g, b), c\right)=U^{2}(d, c)=d \neq g=U^{2}(g, d)=U^{2}\left(g, U^{2}(b, c)\right) .
$$

Corollary 2. (1) in theorem 2 produce an 2-uninorm such that neither conjunctive nor disjunctive 2-uninorm in $U_{k(e, f)}$.

Proposition 2. Let $(L, \leq, 0,1)$ be a bounded lattice, $U^{2} \in U_{k(e, f)}$ such that satisfied conditions of theorem 2. Then, $k$ is absorbing element of $U^{2}$.

Proof. Let $(L, \leq, 0,1)$ be a bounded lattice, $U^{2} \in U_{k(e, f)}$. Then, $U^{2}(k, k)=k$ for $k \in L$ since $k=U^{2}(e, k) \leq U^{2}(k, k) \leq$ $U^{2}(f, k)=k$. It is satisfied that one of $x \leq k, x \geq k$ or $x \| k$ for all $x \in L$. Let $x \leq k$. Then,

$$
k=U_{1}(0, k) \leq U_{1}(x, k)=U^{2}(x, k) \leq U^{2}(k, k)=k
$$

So, $U^{2}(x, k)=k$ for $x \in L$ such that $x \leq k$. Let $x \geq k$. Then,

$$
k=U^{2}(k, k) \leq U^{2}(x, k)=U_{2}(x, k) \leq U_{2}(1, k)=k .
$$

So, $U^{2}(x, k)=k$ for $x \in L$ such that $x \geq k$. Let $x \| k$. Then, $U^{2}(x, k)=k$ for $x \in L$ such that $x \| k$ using (1). Then, it is obtained that $U^{2}(x, k)=k$ for $x \in L$.

Corollary 3. Let $(L, \leq, 0,1)$ be a bounded lattice such that there is at least one element such that incomporable element with $k, U_{1}:[0, k]^{2} \rightarrow[0, k]$ be a disjunctive uninorm with neutral element e and $U_{2}:[k, 1]^{2} \rightarrow[k, 1]$ be a conjunctive uninorm with neutral element $f$. Then, the function $U^{2}: L^{2} \rightarrow L$ as mentioned in theorem 2 is not idempotent 2-uninorm on $L$ even if $U_{1}$ is idempotent uninorm on $[0, k]$ and $U_{2}$ is idempotent uninorm on $[k, 1]$.

Consider the set $U_{k(e, f)}$ of all 2-uninorms on $L$ with the following order:

For $G, H \in U_{k(e, f)}$,

$$
G \leqslant H \Longleftrightarrow G(x, y) \leqslant H(x, y) \text { for all }(x, y) \in L^{2} .
$$

Proposition 3. Let $(L, \leq, 0,1)$ be a bounded lattice. Let take $U_{1}=U_{T_{\wedge}}$ on $[0, k]$ with neutral element e and $U_{2}=U_{S_{\vee}}$ on $[k, 1]$ with neutral element $f$. In these constraints, let call 2 -uninorm in (1) as $U^{*}$. Then, $U^{*}$ satisfies neither $U^{*} \leq F$ nor $U^{*} \geq F$ for every $F \in U_{k(e, f)}$ such that $U^{*} \downarrow[0, k] \neq F \downarrow[0, k]$ and $U^{*} \downarrow[k, 1] \neq F \downarrow[k, 1]$. So, if $U^{*} \downarrow[0, k] \neq F \downarrow[0, k]$ and $U^{*} \downarrow[k, 1] \neq F \downarrow[k, 1], U^{*}$ is incomporable with $F$ for $F \in U_{k(e, f)}$.

Proof. Let assume that $U^{*} \leq F$. Then, $U^{*}(x, y) \leq F(x, y)$ for all $(x, y) \in L^{2}$. So, it has to be satisfied that $U^{*}(x, y)=$ $U_{T_{\wedge}}(x, y) \leq F(x, y)$ for all $(x, y) \in[0, k]^{2}$. It is known that if $F \in U_{k(e, f)}, F \downarrow[0, k]$ is an uninorm on $[0, k]$ with neutral element $e$. This contradict to $U^{*}(x, y)=U_{T_{\wedge}}$ is greatest uninorm on $[0, k]$. Let assume that $U^{*} \geq F$. Then, $U^{*}(x, y) \leq F(x, y)$ for all 
$(x, y) \in L^{2}$. So, it has to be satisfied that $U^{*}(x, y)=U_{S_{V}}(x, y) \geq F(x, y)$ for all $(x, y) \in[k, 1]^{2}$. It is known that if $F \in U_{k(e, f)}$, $F \downarrow[k, 1]$ is an uninorm on $[k, 1]$ with neutral element $f$. This contradict to $U^{*}(x, y)=U_{S_{\vee}}$ is smallest uninorm on $[0, k]$. So, if $U^{*} \downarrow[0, k] \neq F \downarrow[0, k]$ and $U^{*} \downarrow[k, 1] \neq F \downarrow[k, 1], U^{*}$ is incomporable with $F$ for $F \in U_{k(e, f)}$.

\section{Conclusion}

2-Uninorms is generalization of both uninorms and nullnorms. Considering this, it is very important to study 2-uninorms on bounded lattices. 2-uninorms have been characterizated on [0,1] unit reel interval as the point of discontinuity [1]. 2-uninorms have not been studied on bounded lattice yet in our best knowledge. In this paper, if there are $U_{1}$ disjunctive uninorm $[0, k]$ and $U_{2}$ conjunctive uninorm on $[k, 1]$, it is showed that there is way to obtain 2-uninorms on bounded lattices such that $U^{2} \in U_{k(e, f)}$. Moreover, this construction method gives a way to get 2-uninorms on bounded lattice such that neither conjunctive nor disjunctive. Additionally, it is showed that 2-uninorms obtained by this method does not have to be idempotent even if $U_{1}$ and $U_{2}$ are.

\section{Competing interests}

The authors declare that they have no competing interests.

\section{Authors' contributions}

All authors have contributed to all parts of the article. All authors read and approved the final manuscript.

\section{References}

[1] P. Akella, C-sets of n-uninorms, Fuzzy Sets and Systems, 160 (2009), 1-21.

[2] P. Akella, Structure of n-uninorms, Fuzzy Sets and Systems, 158 (2007), 1631-1651.

[3] G. Birkhoff, Lattice Theory, 3 rd edition, Providence, 1967.

[4] P. Drygaś, E. Rak, Distributivity equation in the class of 2-uninorms, Fuzzy Sets and Systems, 2016 (291), 82-97.

[5] Ü. Ertuğrul, F. Karaçal, R. Mesiar, Modified ordinal sums of triangular norms and triangular conorms on bounded lattices, International Journal of Intelligent Systems, 30 (2015) 807-817.

[6] Ü. Ertuğrul, M. N. Kesicioğlu, F. Karaçal, Ordering based on uninorms, Information Sciences, 330 (2016), 315-327.

[7] Ü. Ertuğrul, M. N. Kesicioğlu, F. Karaçal, Ordering based 2-uninorms on bounded lattices, New Trends in Mathematical Sciences, in press.

[8] J. Fodor, R. Yager, and A. Rybalov, Structure of uninorms, Internata. J. Uncertain. Fuzziness Knowledge-Based Systems, 5 (1997), 411-427.

[9] M. Grabisch, J.-L. Marichal, R. Mesiar, E. Pap, Aggregation Functions, Cambridge University Press, 2009.

[10] F. Karaçal, M.A. İnce, R. Mesiar, Nullnorms on bounded lattices, Information Sciences, 325 (2015), 227-236.

[11] F. Karaçal, R. Mesiar, Uninorms on bounded lattices, Fuzzy Sets and Systems, 261 (2015), 33-43.

[12] R. R. Yager, A. Rybalov, Uninorm aggregation operators, Fuzzy Sets and Systems, 80 (1996), 111-120.

[13] R. R. Yager, Uninorms in fuzzy system modelling, Fuzzy Sets and Systems, 122 (2001), 167-175. 